\title{
Anti-EGFR-iRGD recombinant protein conjugated silk fibroin nanoparticles for enhanced tumor targeting and antitumor efficiency
}

This article was published in the following Dove Press journal:

OncoTargets and Therapy

27 May 2016

Number of times this article has been viewed

\author{
Xinyu Bian* \\ Puyuan Wu* \\ Huizi Sha \\ Hanqing Qian \\ Qing Wang \\ Lei Cheng \\ Yang Yang \\ Mi Yang \\ Baorui Liu \\ Comprehensive Cancer Center \\ of Drum-Tower Hospital, Medical \\ School of Nanjing University, Clinical \\ Cancer Institute of Nanjing University, \\ Nanjing, People's Republic of China
}

*These authors contributed equally to this work
Correspondence: Baorui Liu Comprehensive Cancer Center of Drum-Tower Hospital, Medical School of Nanjing University, Clinical Cancer Institute of Nanjing University, Zhongshan Road 32I, Nanjing 210008, People's Republic of China

Tel +862583l05082

Email baoruiliu@nju.edu.cn

\begin{abstract}
In this study, we report a novel kind of targeting with paclitaxel (PTX)-loaded silk fibroin nanoparticles conjugated with iRGD-EGFR nanobody recombinant protein (anti-EGFRiRGD). The new nanoparticles (called A-PTX-SF-NPs) were prepared using the carbodiimidemediated coupling procedure and their characteristics were evaluated. The cellular cytotoxicity and cellular uptake of A-PTX-SF-NPs were also investigated. The results in vivo suggested that NPs conjugated with the recombinant protein exhibited more targeting and anti-neoplastic property in cells with high EGFR expression. In the in vivo antitumor efficacy assay, the A-PTXSF-NPs group showed slower tumor growth and smaller tumor volumes than PTX-SF-NPs in a HeLa xenograft mouse model. A real-time near-infrared fluorescence imaging study showed that A-PTX-SF-NPs could target the tumor more effectively. These results suggest that the anticancer activity and tumor targeting of A-PTX-SF-NPs were superior to those of PTX-SFNPs and may have the potential to be used for targeted delivery for tumor therapies.
\end{abstract}

Keywords: EGFR, nanobody, iRGD, recombinant protein, targeting drug carriers, antitumor efficiency

\section{Introduction}

Most current chemotherapy drugs do not sufficiently distinguish between cancerous and normal cells, leading to systemic adverse effects and dose-limiting toxicity. Moreover, rapid elimination of anticancer drugs reduces the curative effect and requires the administration of the drug in large quantities, which results in poor response, high cost, and undesirable toxicity. ${ }^{1}$ Attention is now being focused on killing cancer cells by more specific targeting while sparing normal cells. To achieve these goals, one of the methods is the development of novel delivery systems for both existing and new drugs. Nanoparticles (NPs), the advanced, sustained drug delivery systems, are making a significant contribution to the improvement in oncology therapy, such as enhancing the anticancer efficacy and reducing drug toxicity. ${ }^{2}$ In addition, it has been confirmed that a tumor can take up NPs passively through enhanced permeability and retention (EPR). The fundamental features of EPR physiology are hyperpermeable tumor vasculature allowing enhanced permeability of large particles, which are large enough to avoid renal clearance into the interstitial space of the tumor. Meanwhile, the impaired lymphatic drainage of tumor limits the clearance of these particles and causes their enhanced retention. ${ }^{3}$

However, many clinical outcomes have indicated that EPR is not as reliable to ensure tumor targeting of NPs as previously proved by preclinical studies, which 
may be attributed to the complex tumor microenvironment. ${ }^{4}$ To overcome the limitation of passive targeting, a variety of methods have been used to ensure that NPs acquire active tumor targeting ability; one of them is to couple NPs with various biomolecules, such as monoclonal antibodies, peptides, aptamers, etc. These active targeting moieties are capable of reducing off-target effects and improving the bioavailability of the NPs. ${ }^{5}$

Epidermal growth factor receptor (EGFR), which is overexpressed in multiple human solid tumors, is closely related to the prognosis and response to chemotherapy. Thus, it has been a promising target for therapy and a mediator for targeted delivery. ${ }^{6,7}$ It has been reported that the tumortargeting and antitumor effect of NPs for cells with high EGFR expression could be improved evidently when NPs are conjugated with EGFR inhibitors. ${ }^{8,9}$ Several studies also have indicated that EGFR inhibitors have synergistic effects with chemotherapy drugs, such as paclitaxel (PTX), pemetrexed, and irinotecan. ${ }^{10-12}$ iRGD, a nine-unit, cyclic, tumor-homing peptide containing the RGD sequence, has been confirmed to increase vascular and tissue permeability in a tumor-specific and neuropilin-1-dependent manner. This peptide activates the CendR pathway specifically via its specific recognition of $\alpha v \beta 3$ and $\alpha v \beta 5$ integrins, which are highly expressed in parenchyma cells and vessels in a variety of solid tumors. ${ }^{13}$ Thus, when drug carriers are modified with iRGD, their abilities of tumor targeting, tissue penetration, and tumor suppression can be improved significantly. ${ }^{14}$ PTX, a liposoluble anticancer drug, has been demonstrated to show anti-neoplastic activity against various types of solid tumors including gastric and cervical cancer. ${ }^{15,16}$ To improve its solubility, it is formulated in a 1:1 blend of Cremophor EL/ absolute ethanol as Taxol, which often causes hypersensitivity reaction and neurotoxicity. ${ }^{17}$ In order to reduce these adverse side effects, several strategies have been developed to establish PTX-containing NPs with polymers, liposomes, and albumins. These NP formulations are capable of enhancing the safety, pharmacokinetic profiles, and bioavailability of PTX. ${ }^{18}$

Previously, we have constructed a recombinant protein named anti-EGFR-iRGD consisting of an anti-EGFR VHH (the variable domain from the heavy chain of the antibody) fused to iRGD. The recombinant protein dually targets EGFR and $\alpha v \beta 3 / \alpha v \beta 5$ integrins. It shows anticancer effect in vitro and in vivo and has synergistic effects with PTX. ${ }^{19}$ We also have developed a novel kind of PTX-containing NPs with nontoxic materials using a facile method. The NPs were formed in an aqueous solution at room temperature by self-assembling silk fibroin (SF) protein, and their main characteristics were evaluated. The drug loading content was
$10 \% \pm 2 \%$ and drug encapsulation efficiency was $52 \% \pm 2 \% .{ }^{20}$ In this study, we conjugate anti-EGFR-iRGD to the paclitaxel-silk fibroin NPs (PTX-SF-NPs) and investigate their tumor-targeting and anticancer activity.

\section{Materials and methods Materials}

Cocoons of Bombyx mori silkworm were obtained from Jiangsu Province, People's Republic of China. PTX was purchased from Jiangsu Yew Pharmaceutical Co (Jiangsu, People's Republic of China). Anti-EGFR-iRGD was a kind gift from Doctor Sha (The Comprehensive Cancer Center of DrumTower Hospital, Medical School of Nanjing University \& Clinical Cancer Institute of Nanjing University).

1-Ethyl-3-(3-dimethylaminopropyl)-carbodiimide and $\mathrm{N}$-hydroxylsuccinimide were obtained from Acros (NJ, USA). 3-(4,5-Dimethylthiazol-2-yl)-2,5-diphenyltetrazolium bromide (MTT) was purchased from Sigma-Aldrich Co., (St Louis, MO, USA). RPMI 1640 (Thermo Fisher Scientific, Waltham, MA, USA) and calf blood serum (Lanzhou Minhai Bioengineering Co., Ltd., Beijing, People's Republic of China) were used as received. Deionized (DI) water, produced by a Millipore water system, was utilized throughout all experiments. EGFR primary antibody was from Cell Signaling Technology (Danvers, MA, USA), and secondary antibody conjugated to horseradish peroxidase was from Abcam (Cambridge, MA, USA). Coumarin-6, NIR-797-isothiocyanate, and fluorescein isothiocyanate (FITC) were purchased from Sigma Chemicals (Perth, WA, Australia). All other reagents were of analytical grade and used without further purification. Human cervical cancer cell line HeLa and human gastric adenocarcinoma cell line MKN-45 were obtained from Shanghai Institute of Biochemistry and Cell Biology (Shanghai, People's Republic of China). No ethics statement was required from the Ethics Committee of Nanjing Drum Tower Hospital for the use of these cell lines.

\section{SF purification and sterilization}

Aqueous stock solutions of SF were prepared as previously reported. ${ }^{20}$ Briefly, cocoons of B. mori silkworm were boiled for 30 minutes in $0.5 \%$ sodium carbonate twice after chopping and rinsing thoroughly with DI water. Then the extractive was dried in vacuum at $60^{\circ} \mathrm{C}$ and dissolved in a ternary solution (molar ratio $\mathrm{CaCl}_{2} / \mathrm{H}_{2} \mathrm{O} / \mathrm{CH}_{3} \mathrm{CH}_{2} \mathrm{OH}=1: 8: 2$ ) at $60^{\circ} \mathrm{C}$ for 5 hours. This solution was dialyzed against DI water with a dialysis bag (MWCO 10,000 Da) for 3 days to remove the inorganic salts and ethanol. After centrifuging the solution at $12,000 \mathrm{rpm}$ for 20 minutes, the supernatant was collected and filtered with a $0.22 \mu \mathrm{m}$ filter to remove microorganisms. The final concentration of SF was determined with a BCA kit. 


\section{Preparation of PTX-SF-NPs}

PTX-SF-NPs were prepared as described previously. ${ }^{20}$ Briefly, $5 \mathrm{mg}$ of PTX was dissolved in $0.4 \mathrm{~mL}$ of absolute ethanol and added dropwise to $5 \mathrm{~mL}$ of $0.5 \%(\mathrm{w} / \mathrm{v}) \mathrm{SF}$ solution with gentle stirring. After stirring for 5 minutes, the resulting suspension was centrifuged at $12,000 \mathrm{rpm}$ for 20 minutes. Then the sediment was washed twice with DI water by centrifugation and resuspended in PBS (phosphate buffered saline) $(\mathrm{pH}=7.2)$ with an ultrasound processor SONICS Vibra-cell VCX130 (Sonics \& Materials Inc., Newtown, CT, USA) at $20 \%$ amplitude for $2-3$ seconds.

\section{Conjugation of anti-EGFR-iRGD to PTX-SF-NPs}

The PTX-SF-NPs were prepared as described earlier. The only difference was that the sedimentation was resuspended in MES buffer ( $\mathrm{pH}=5.2)$ after rinsing. Four milligrams of 1-ethyl-3-(3-dimethylaminopropyl)-carbodiimide and $11 \mathrm{mg}$ of $N$-hydroxylsuccinimide were added to $1 \mathrm{~mL}$ of the $0.5 \%(\mathrm{w} / \mathrm{v}) \mathrm{NP}$ suspension. The mixture was stirred and incubated for 30 minutes at room temperature. Then, $1 \mathrm{mg}$ of the anti-EGFR-iRGD was added to the suspension and the $\mathrm{pH}$ was brought close to 7.0 by adding PBS buffer $(\mathrm{pH}=7.2)$ to the reaction system. The reaction was continued with mild stirring at $4^{\circ} \mathrm{C}$ for 4 hours and was terminated by adding $1 \mathrm{mg} / \mathrm{mL}$ hydroxylamine hydrochloride to the solution. The suspension was centrifuged at $12,000 \mathrm{rpm}$ for 20 minutes and the pellet was washed twice with PBS buffer ( $\mathrm{pH}=7.2$ ). The resulting sediment (A-PTXSF-NPs) was resuspended in PBS buffer $(\mathrm{pH}=7.2)$ by ultrasonic dispersion.

\section{Characterization of PTX-SF-NPs and A-PTX-SF-NPs}

Particle size and zeta potential of these two NPs were measured using a Brookhaven BI-900AT instrument/zeta potential analyzer (Brookhaven Instruments Corporation, Holtsville, NY, USA). Fourier transform infrared (FTIR) spectra were recorded using a Nicolet iS10 FTIR spectrometer (Thermo Scientific, Swedesboro, NJ, USA). Morphology studies of the NPs were carried out using transmission electron microscopy (TEM) (JEM-100s; JEOL, Tokyo, Japan).

\section{Recombinant protein conjugation efficiency on A-PTX-SF-NPs}

To quantitatively determine the conjugation efficiency, a fluorescence RF-5301PC spectrometer (Shimadzu, Japan) was used. Generally, the recombinant protein was conjugated with FITC at the amine groups of lysine. The labeled protein was dialyzed against PBS to remove any unconjugated FITC. FITC-labeled A-PTX-SF-NPs were prepared with the same procedure except that antibody-EGFR-iRGD, instead of FITC, was labeled. The resuspended, FITC-labeled A-PTXSF-NPs supernatant, after reaction and washing with DI water or PBS buffer by centrifugation, was collected for fluorescence measurement with excitation and emission wavelengths of $490 \mathrm{~nm}$ and $525 \mathrm{~nm}$, respectively. Standard curve was obtained corresponding to the fluorescence intensity of different concentrations of original FITC-labeled anti-EGFR-iRGD solution. After quantifying the amount of unconjugated recombinant protein in the supernatant, the conjugation efficiency ( $\mathrm{CE} \%$ ) was finally calculated by the following formula:

$$
\mathrm{CE} \%=\frac{\begin{array}{c}
\text { Total amount of anti-EGFR-iRGD } \\
\text { added }- \text { Amount of anti-EGRF-iRGD } \\
\text { left in the supernatant }
\end{array}}{\text { Total amount of anti-EGFR-iRGD added }} \times 100 \%
$$

\section{Western blot assay}

The protein expression level of EGFR was determined using a standard protocol. Briefly, cell extracts of HeLa and MKN-45 were collected by using the PIPA lysate (P0013C, Beyotime). The collections were prepared with loading buffer and separated by SDS-PAGE at a constant voltage and electrotransfered onto polyvinylidene difluoride (PVDF) membranes (EMD Millipore, Billerica, MA, USA). The membranes were then blocked at room temperature for $\sim 1$ hour with shaking and incubated with a primary antibody overnight at $4^{\circ} \mathrm{C}$. After rinsing with TTBS for three times, the membranes were incubated with a secondary antibody for 1 hour. The blots were visualized using an enhanced chemiluminescence method after washing three times.

\section{In vitro cytotoxity}

MTT assay was carried out to test the in vitro cytotoxity of PTX-SF-NPs and A-PTX-SF-NPs against the two cancer lines HeLa and MKN-45. Cells (5,000 cells per well) were seeded in 96-well plates with RPMI 1640 medium supplemented with $10 \%$ calf serum. After incubating at $37^{\circ} \mathrm{C}$ with $5 \% \mathrm{CO}_{2}$ for 24 hours, the cells were then exposed to various concentrations of PTX-SF-NPs and A-PTX-SFNPs for 48 hours. Then, $20 \mu \mathrm{L}$ of $5 \mathrm{mg} / \mathrm{mL}$ MTT solution was added to each well, and incubation was carried out for another 4 hours. The medium was discharged, and $150 \mu \mathrm{L}$ DMSO per well was added to dissolve the formazan crystals. 
The absorption at $490 \mathrm{~nm}$ was measured by an ELISA reader (ELX800 Biotek), and the cell viabilities were calculated by the following formula:

$$
\text { Cell viability }=\frac{\text { Abs (sample) }- \text { Abs (background })}{\text { Abs (control) }- \text { Abs (background })} \times 100 \%
$$

\section{Cellular uptake of NPs in vitro}

For in vitro cellular uptake study, coumarin-6-loaded NPs were prepared with the same procedure except for coumarin-6 replacing PTX as the hydrophobic core. HeLa cells in the logarithmic growth phase were seeded in six-well plates ( $10^{5}$ cells per well) with RPMI 1640 medium supplemented with $10 \%$ calf serum. After incubating at $37^{\circ} \mathrm{C}$ with $5 \%$ $\mathrm{CO}_{2}$ for 24 hours, coumarin-6-SF-NPs and A-coumarin-6SF-NPs were added into the wells (equal to $5 \mu \mathrm{g} / \mathrm{mL}$ in the medium) and incubated for 1 hour each. After washing with ice-cold PBS three times, the fluorescent signals of cells were observed with a fluorescence microscope (Olympus Corporation, Tokyo, Japan). The mean optical density was quantified with ImageJ $1.48 \mathrm{~V}$ (NIH, USA).

\section{In vivo antitumor efficacy}

The in vivo antitumor efficacy was evaluated by using HeLa tumor-bearing nude mice. All animal experiments were approved by the Animal Care Committee, Drum Tower Hospital, Nanjing, People's Republic of China. All animal-handling procedures were performed in compliance with guidelines set by the Animal Care Committee, Drum Tower Hospital. BALB/c (nu/nu) mice (4 weeks, 18-22 g, male) were subcutaneously injected with HeLa cells $\left(5 \times 10^{6}\right.$ cells per mouse $)$. Ten days after cell injections, the tumor-bearing nude mice were randomly divided into three groups (five mice per group); the day of randomization was designated as Day 1. Group 1 was treated with normal saline (NS) as control, group 2 was treated with PTX-SF-NPs $(75 \mathrm{mg} / \mathrm{kg})$, and group 3 was treated with A-PTX-SF-NPs $(75 \mathrm{mg} / \mathrm{kg}$ PTX-SFNPs equiv). All treatments were intravenously administrated via a tail vein every 4 days three times. Throughout the study, size of the tumors was measured with a caliper every other day and the volume was calculated as $\mathrm{V}=\mathrm{D} \times \mathrm{d}^{2} / 2$, where $\mathrm{d}$ and $\mathrm{D}$ are the shortest and the longest diameter of the tumor in millimeters, respectively. In order to reduce the impact of initial tumor volume differences among different groups, relative tumor volumes were calculated by the following formula:

$$
\text { Relative tumor volumes }=\frac{\mathrm{V}}{\mathrm{V}_{0}}
$$

In Equation 3, V represents the absolute tumor volume, and $\mathrm{V}_{0}$ represents the average tumor volume of the group on Day 1. On Day 14, the mice were sacrificed, and their heart, lungs, liver, spleen, and kidneys were collected for hematoxylin and eosin staining to assess the systemic toxicity.

\section{In vivo imaging}

To investigate the tumor targeting and accumulation of NPs in vivo, NIR-797 isothiocyanate was used as an imaging agent. First, $3 \mathrm{mg}$ of NIR-797 isothiocyanate dissolved in $0.5 \mathrm{~mL}$ of anhydrous DMSO was added to $10 \mathrm{~mL}$ of $0.5 \%$ (w/v) SF solution under gentle stirring in the dark for 12 hours. Unreacted NIR-797 was removed by dialysis against DI water with a dialysis bag (MWCO 10,000 Da) for 2 days. Then the NIR-797-labeled SF was used to prepare NPs with the same produce as described in the previous section. HeLa-bearing mice described earlier were injected with NIR-797-labeled PTX-SF-NPs and A-PTX-SF-NPs via a tail vein. The NIR fluorescence images were acquired, and the signal intensity was measured with a Maestro EX in vivo fluorescence imaging system (CRi, Inc., Hopkinton, MA, USA). Scans were performed at different time points post administration. To evaluate the accumulation and biodistribution of NPs in vivo, the relative signal intensities were calculated by the following formula:

$$
\underset{\text { intensity }}{\text { Relative signal }}=\frac{\text { Average signal tumor }}{\text { Average signal liver }}
$$

At the end of test, the tumor-bearing mice were sacrificed, and the tumor, heart, liver, spleen, lungs, and kidneys were collected for isolated imaging to estimate the biodistribution of NPs in the organs.

\section{Results \\ Characterization of PTX-SF-NPs and A-PTX-SF-NPs}

In this work, we used ethanol as the PTX solvent to optimize the preparation technology according to our previous work. ${ }^{20}$ The mean size of PTX-SF-NPs and A-PTX-SF-NPs was $137.8 \pm 13.1 \mathrm{~nm}$ and $186.0 \pm 19.7 \mathrm{~nm}$, respectively, both with a narrow distribution (polydispersity index [PDI] $0.22 \pm 0.04$ and $0.24 \pm 0.05$, respectively). The presence of recombinant protein on the NPs' surface increased the particle size (Table 1). Both the NPs were mostly spherical and well dispersed according to TEM. TEM analysis showed that the diameter of A-PTX-SF-NPs was greater than that of PTX-SF-NPs (Figure 1), which was consistent with the result measured by dynamic light scattering (DLS). The zeta 
Table I Size, PDI, zeta potential, and conjugation efficiency of the NPs

\begin{tabular}{lll}
\hline & PTX-SF-NPs & A-PTX-SF-NPs \\
\hline Mean size, $\mathrm{nm}$ & $137.8 \pm 13.1$ & $186.0 \pm 19.7$ \\
PDI & $0.22 \pm 0.04$ & $0.24 \pm 0.05$ \\
Zeta potential, $\mathrm{mV}$ & $-8.13 \pm 1.59$ & $-12.08 \pm 0.82$ \\
Conjugation efficiency, \% & - & $76.27 \pm 12.29$ \\
\hline
\end{tabular}

Note: Data presented as mean \pm standard deviation.

Abbreviations: PDI, polydispersity index; NPs, nanoparticles; PTX, paclitaxel; SF, silk fibroin.

potential of PTX-SF-NPs was $8.13 \pm 1.59$, whereas that of A-PTX-SF-NPs was $-12.08 \pm 0.82$.

The FTIR spectra of PTX-SF-NPs and A-PTX-SF-NPs were similar. In addition, for A-PTX-SF-NPs, the intensity of the main absorption bands at $1,646 \mathrm{~cm}^{-1}(\mathrm{C}=\mathrm{O})$ and 3,068-3,651 $\mathrm{cm}^{-1}$ $(\mathrm{O}-\mathrm{H})$ increased significantly (Figure $2 \mathrm{~A})$. This change indicated that A-PTX-SF-NPs contained more carboxy groups.

Spectrofluorometry was used to identify the existence of anti-EGFR-iRGD on A-PTX-SF-NPs and to determine the conjugation efficiency. The fluorescence of FITC could be detected in A-PTX-SF-NPs and the supernatant (Figure 2B), and the conjugation efficiency was $76.27 \% \pm 12.29 \%$ (Table 1). The fluctuation of conjugation efficiency may be attributed to the amount of carboxyl groups on the surface of the NPs.

\section{EGFR level in the two cell lines}

The expression levels of EGFR in the two cell lines were measured by Western blot. Compared with that in HeLa, the EGFR levels in MKN-45 cells could not even be detected (Figure 3A).

\section{In vitro cytotoxicity and cellular uptake of NPs}

In vitro cytotoxicity of NPs was evaluated using the MTT assay in HeLa and MKN-45 cells. Both cancer cells were treated with increasing doses of PTX-SF-NPs and A-PTXSF-NPs. A dose-dependent cytotoxicity was observed for both PTX-SF-NPs and A-PTX-SF-NPs in HeLa and MKN-45 cells. A-PTX-SF-NPs exhibited significantly increased cytotoxicity compared to PTX-SF-NPs $(P<0.05)$ in HeLa cells, except at the concentration of $16 \mu \mathrm{g} / \mathrm{mL}(P=0.051)$ (Figure $3 \mathrm{~B})$. Meanwhile, in the MKN-45 cell line with poor EGFR expression, although the cell viability of the A-PTX-SF-NPs group was lower than that of the PTX-SF-NPs group, no significant difference was found (Figure 3C). In the cellular uptake assay, coumarin-6-loaded NPs were used to co-incubate with HeLa cells for 1 hour at $37^{\circ} \mathrm{C}$. It can be seen that the fluorescence of A-coumarin-6-SF-NPs was significantly stronger than that of coumarin-6-SF-NPs (Figure 4A and B). The mean optical density of the PTX-SF-NPs group and the A-PTXSF-NPs group was $6.56 \pm 0.37$ and $12.63 \pm 1.91$, respectively (Figure 4C). These results showed that A-Coumarin-6-SF-NPs were more targetable to cells with high EGFR expression, consistent with our in vitro cytotoxicity result.

\section{In vivo antitumor activity}

The in vivo antitumor efficacy of PTX-SF-NPs and A-PTXSF-NPs was evaluated in the nude mice bearing subcutaneous inoculated HeLa tumors. The treatments were administrated via a tail vein with NS, PTX-SF-NPs, and A-PTX-SF-NPs, respectively, and repeated every 4 days three times a day. As is shown in Figure 5A, the relative tumor volumes for the NS group were the largest among all the groups at all measuring time points $(P<0.01)$. The A-PTX-SF-NPs group showed slower tumor growth and smaller relative tumor volumes than the PTX-SF-NPs group and the differences were highly significant $(P<0.05)$ except on Day $4(P=0.068)$. On Day 13, the relative tumor volumes were $1.50 \pm 0.30$ for the A-PTX-SF-NPs group, 3.35 \pm 0.61 for the PTX-SF-NPs group, and $8.23 \pm 1.99$ for the NS group.
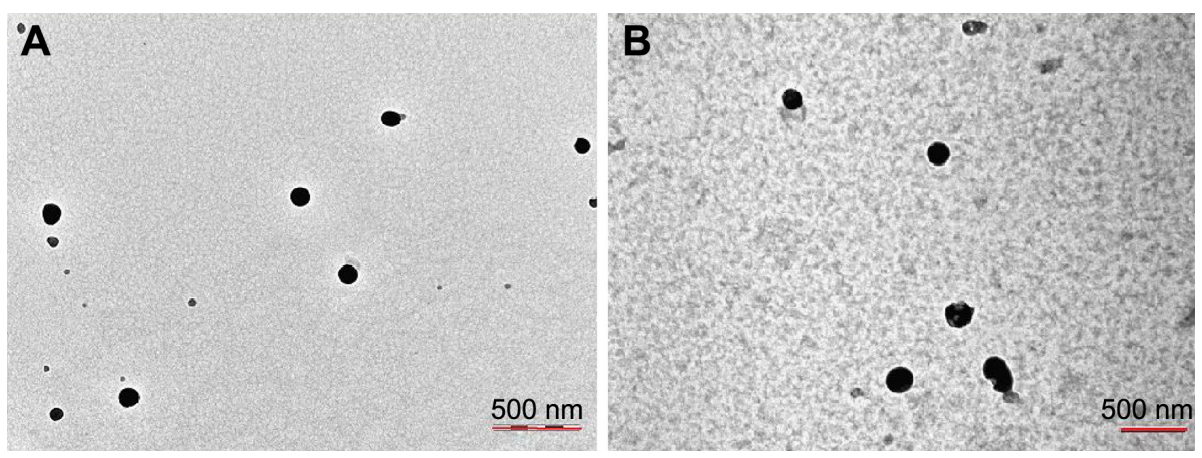

Figure I (A) TEM image of PTX-SF-NPs. (B) TEM image of A-PTX-SF-NPs.

Note: Magnification, $\times 40000$.

Abbreviations: TEM, transmission electron microscopy; PTX, paclitaxel; SF, silk fibroin; NPs, nanoparticles. 

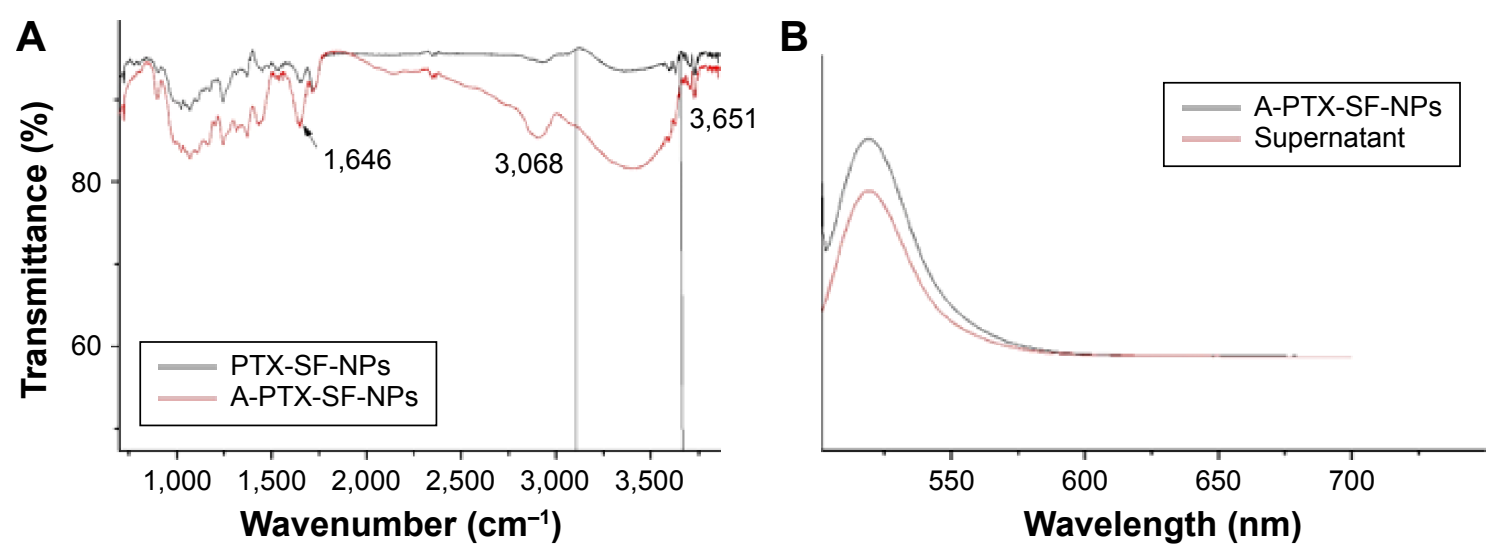

Figure 2 (A) FTIR of PTX-SF-NPs and A-PTX-SF-NPs. (B) Fluorescence spectra of A-PTX-SF-NPs and supernatant after reaction. Abbreviations: FTIR, Fourier transform infrared; PTX, paclitaxel; SF, silk fibroin; NPs, nanoparticles.

In addition, to evaluate the acute systemic toxicity of PTX-SF-NPs and A-PTX-SF-NPs, the mice were sacrificed on Day 14 and pathological studies of important organs in each group were carried out, including the heart, lungs, liver, spleen, and kidneys. No obvious morphological changes of the organs were found, indicating good biocompatibility and in vivo safety of both NPs (Figure 5B).

\section{In vivo imaging}

The fate and accumulation of A-PTX-SF-NPs and PTX-SFNPs in vivo were monitored via real-time NIR fluorescence imaging. As is shown in Figure 6A and B, during the initial 1 hour post injection, both A-PTX-SF-NPs and PTX-SFNPs accumulated in the liver and intestine. At 4 hours post injection, fluorescence signal appeared at the tumor site for both A-PTX-SF-NPs and PTX-SF-NPs. The fluorescence signal in the tumor of PTX-SF-NPs was weaker than that of A-PTX-SF-NPs except at 4 hours post injection (Figure 6C). Both NPs retained a strong fluorescence signal at the tumor site until the test ended. It took 144 hours to reach the maximum fluorescence intensity for PTX-SF-NPs, while it took only 24 hours for A-PTX-SF-NPs (Figure 6D). In contrast, the fluorescence intensity of both NPs in the

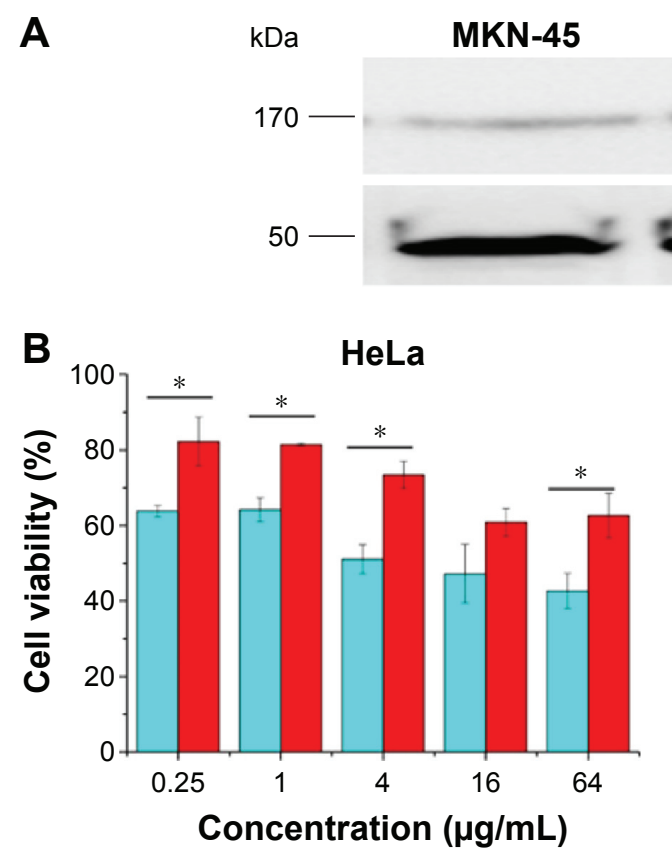

HeLa

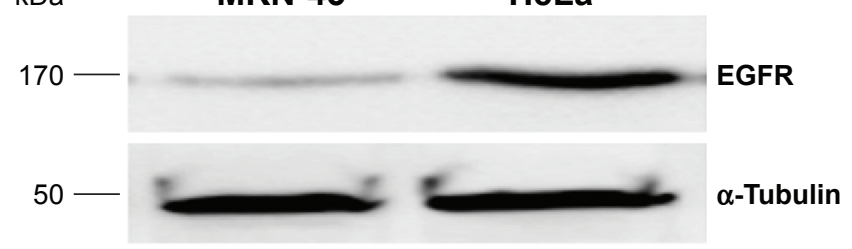

C

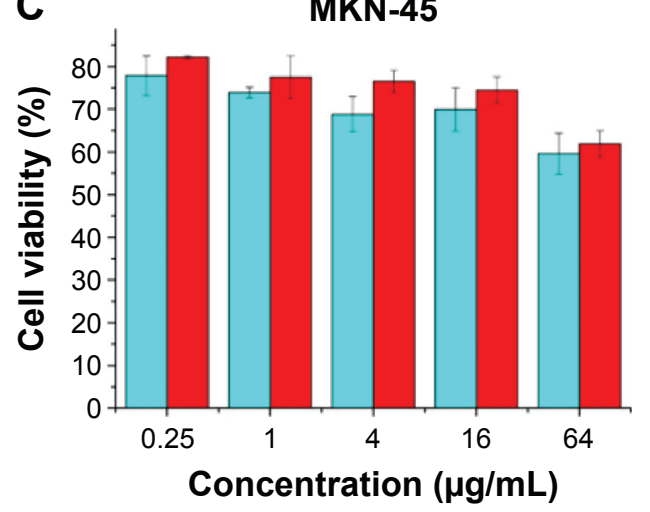

$\square$ A-PTX-SF-NPs

PTX-SF-NPs

Figure 3 The cytotoxicity of PTX-SF-NPS and A-PTX-SF-NPS against tumor cells with different EGFR expression.

Notes: (A) EGFR levels in HeLa and MKN-45 cells. (B) In vitro cytotoxicity of PTX-SF-NPs and A-PTX-SF-NPs against Hela cell lines. (C) In vitro cytotoxicity of PTX-SF-NPs and A-PTX-SF-NPs against MKN-45 cell lines. Data are represented as mean $\pm S D$ and cover relative values, $n=3$. Statistical analyses were performed with Student's $t$-test, $* P<0.05$. Abbreviations: EGFR, epidermal growth factor receptor; PTX, paclitaxel; SF, silk fibroin; NPs, nanoparticles. 

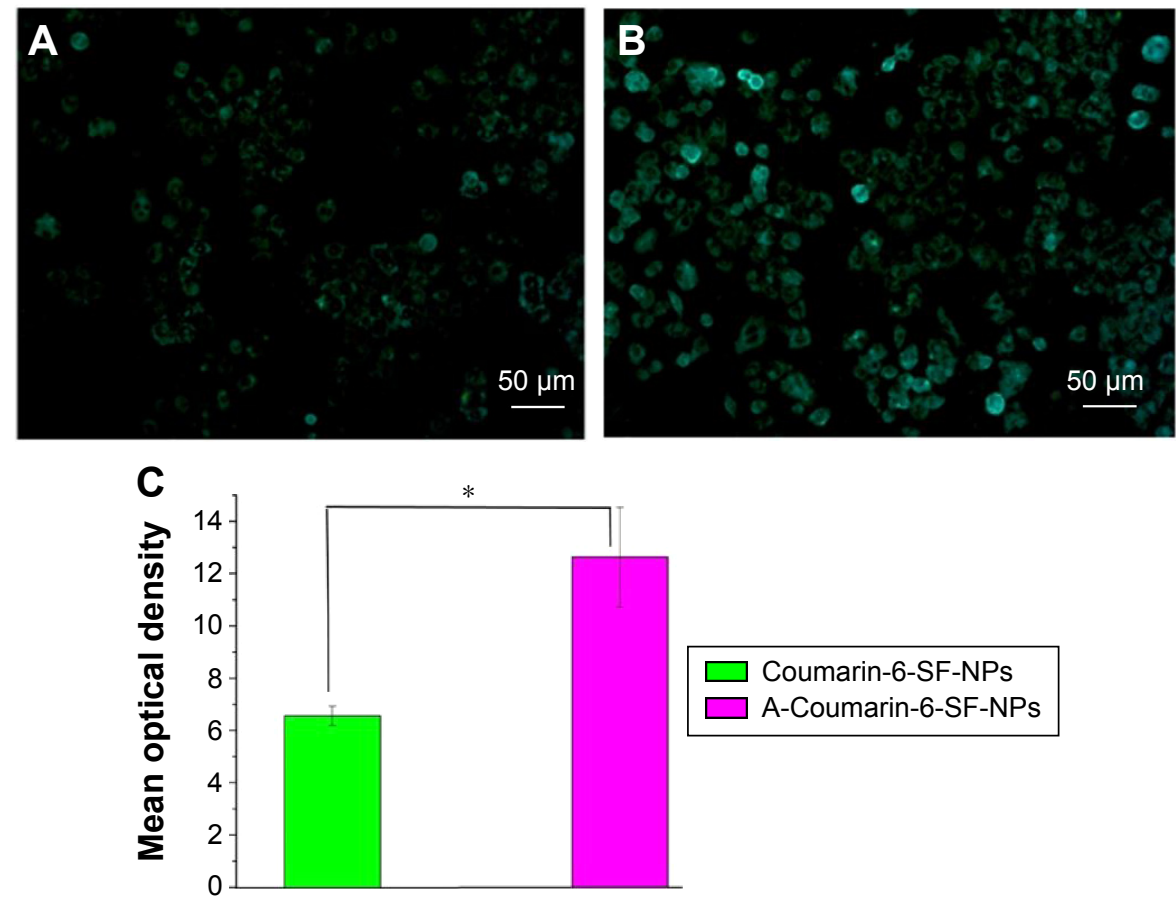

Figure 4 The fluorescence microscopic images of Hela cells after incubation with Coumarin-6-SF-NPs and A-Coumarin-6-SF-NPs.

Notes: Cellular uptake of Coumarin-6-SF-NPs (A) and A-Coumarin-6-SF-NPs (B) by HeLa cells. (C) The mean optical density of Coumarin-6-SF-NPs and A-Coumarin-6SF-NPs. All data were expressed as the mean \pm standard, $n=3$. Data represent the means of three independent experiments. Statistical analyses were performed using the one-way ANOVA. $* P<0.05$. Magnification, $\times 200$.

Abbreviations: SF, silk fibroin; NPs, nanoparticles; ANOVA, analysis of variance.

liver decreased relatively rapidly. It was also noticed that the fluorescence signal of PTX-SF-NPs was similar to that of A-PTX-SF-NPs in the liver except at 4 hours post injection (Figure 6B). The relative intensity of A-PTX-SF-NPs and PTX-SF-NPs was $>1$ after 12 hours and 96 hours post injection, which meant that more NPs accumulated in the tumor than in the liver (Figure 6E). This result demonstrated that both groups of NPs have prolonged circulation time, but A-PTX-SF-NPs have better tumor-targeting ability than PTX-SF-NPs.

Finally, the mice were sacrificed at 168 hours post injection, and ex vivo fluorescence intensity images were obtained for the tumor tissue as well as other major organs such as the heart, liver, spleen, lungs, and kidneys. As shown in Figure 6F, at 168 hours post injection, strong fluorescence was still observed in the tumor tissue and liver for both
A

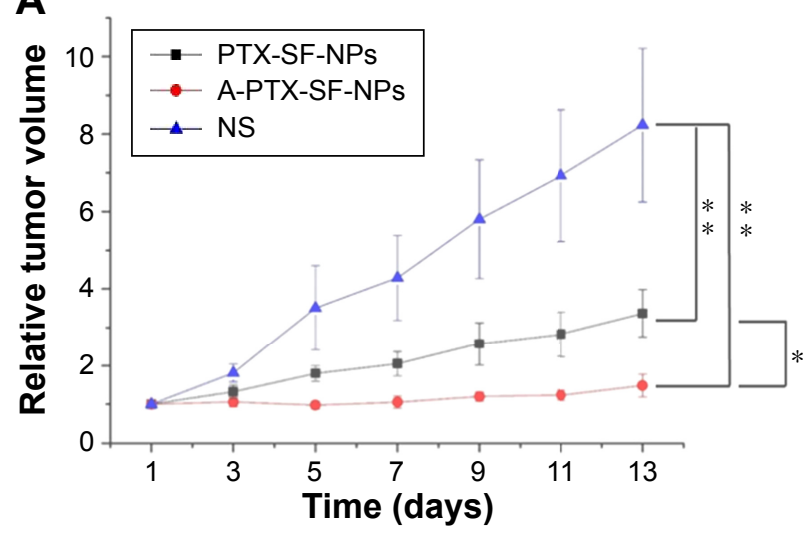

B

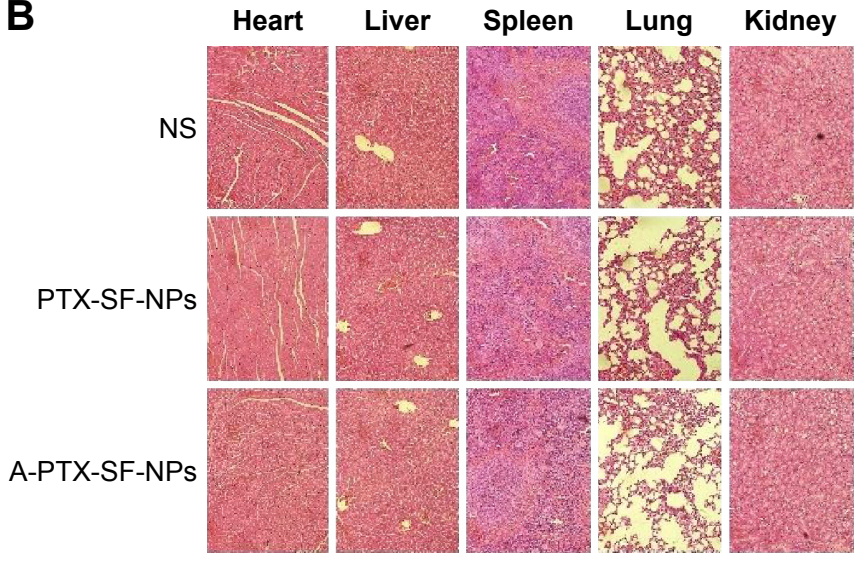

Figure 5 The antitumor effect and systemic toxicity of PTX-SF-NPs and A-PTX-SF-NPs in vivo.

Notes: (A) Relative tumor volumes during treatment of HeLa tumor-bearing mice that received different treatments. Data are represented as mean $\pm S D(n=5)$. $* P<0.05$ and $* * P<0.01$. (B) Main organs (heart, liver, spleen, lung and kidney) were dissected for H\&E staining on the I4th day after treatment. Tissue alterations of only few inflammatory cells infiltrating in liver and spleen could be seen, while no significant abnormal damage were observed.

Abbreviations: NS, normal saline; PTX, paclitaxel; SF, silk fibroin; NPs, nanoparticles; H\&E, hematoxylin and eosin. 


\section{A}
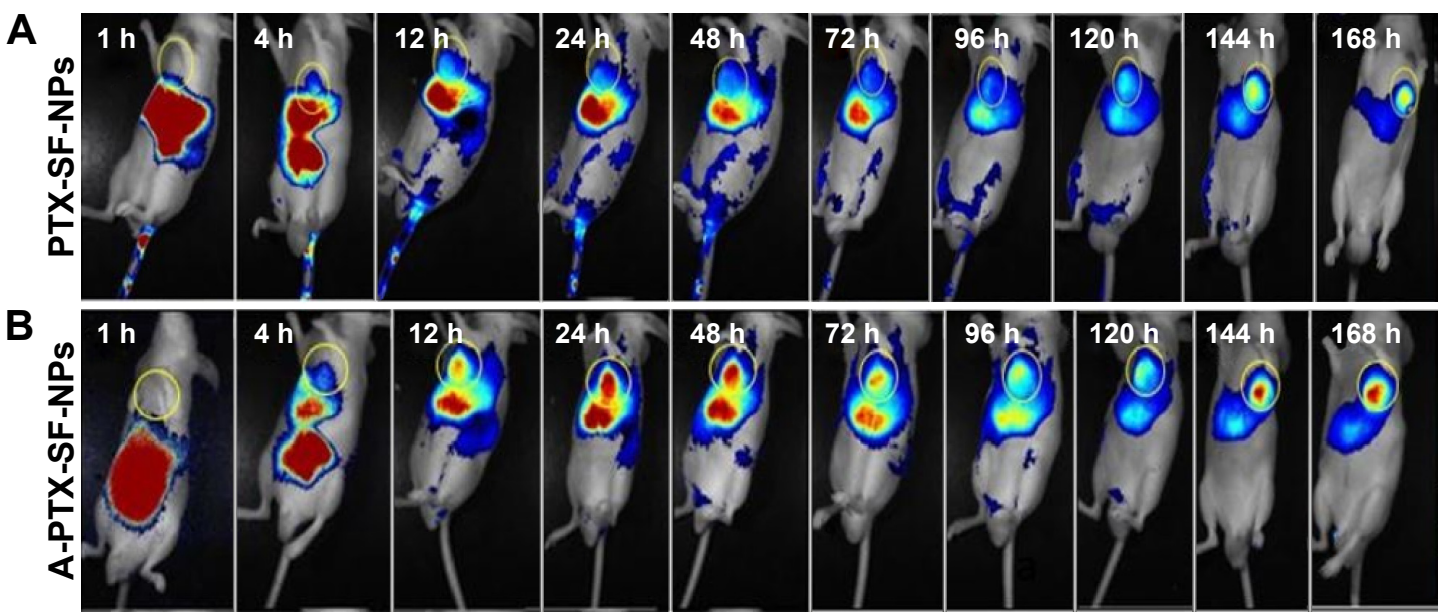

C

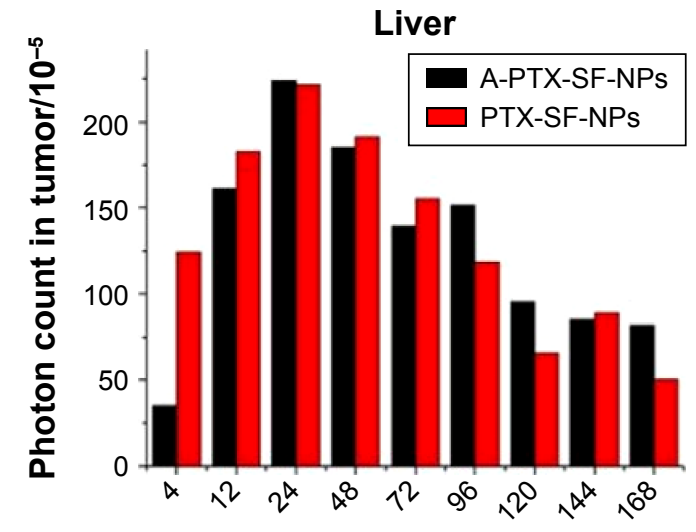

$D_{0}$

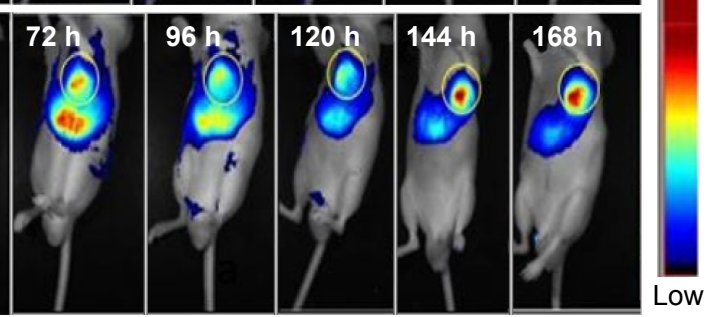

Time (hours)
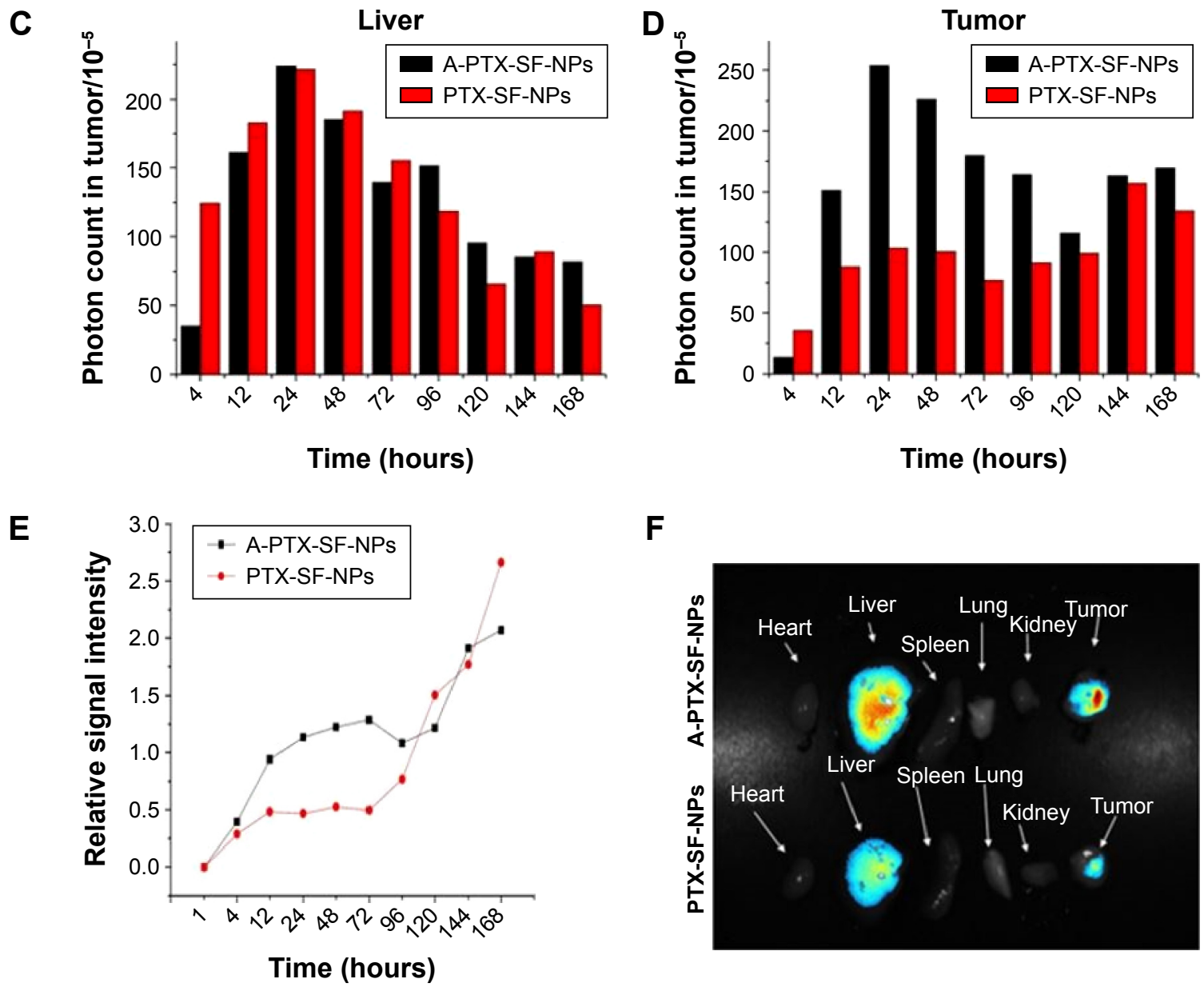

$\mathbf{F}$

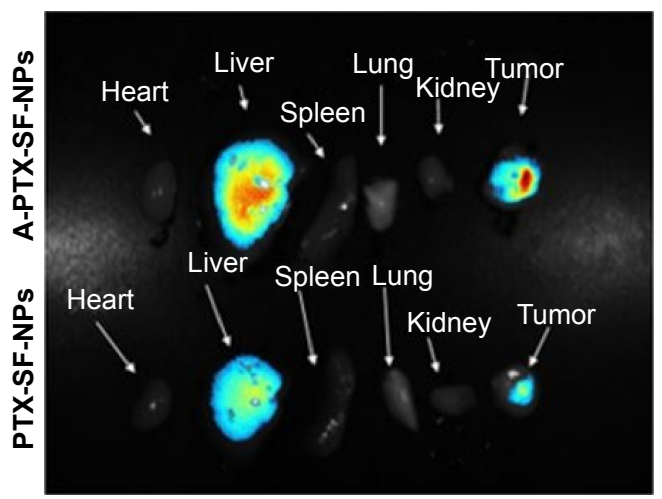

Figure 6 The NIRF images of HeLa tumor-bearing mice following intravenous injection of NIR-797 labeled PTX-SF-NPs (A) and A-PTX-SF-NPs (B). Absolute signal intensity of NPs in liver (C) and tumor (D). (E) Relative signal intensity of PTX-SF-NPs and A-PTX-SF-NPs. (F) NIRF images of various organs at I68 h post-administration with PTX-SF-NPs and A-PTX-SF-NPs.

Abbreviations: PTX, paclitaxel; SF, silk fibroin; NPs, nanoparticles; NIRF, near infrared fluorescence.

A-PTX-SF-NPs and PTX-SF-NPs, while other tissues showed negligible fluorescence signal. Furthermore, it was noticed that for A-PTX-SF-NPs, the fluorescence signal in the tumor was much higher than that in the liver, while PTXSF-NPs' signal in the tumor was similar to that in the liver.

\section{Discussion}

In recent decades, active tumor-targeting drug delivery systems have attracted particular interest in cancer treatment with the capability to facilitate drug delivery to tumor cells. Conjugating a drug with a tumor-specific molecule is one of the most 
commonly used approaches to realize the objective. ${ }^{21}$ In previous work, we developed a recombinant protein named anti-EGFRiRGD based on anti-EGFR VHH and a tumor-penetrating peptide, and demonstrated its targeting integrin and EGFR, inhibiting EGFR Tyr1172 phosphorylation and with high penetration ability. ${ }^{19}$ In this study, we investigated further whether anti-EGFR-iRGD was capable of enhancing the tumor-specific targeting of NPs when conjugated with them. We selected PTXSF-NPs as the NPs because of their simple preparation method and significant anticancer activity. Meanwhile, there was a fair amount of carboxyl groups on the surface of PTX-SF-NPs, which could be used to link with the recombinant protein via carbodiimide chemistry. ${ }^{22}$ Since the conjugation efficiency of anti-EGFR-iRGD and anti-EGFR-iRGD singly conjugated NPs was different and incomparable (data not shown), we just chose anti-EGFR-iRGD as the targeting ligand.

Our results showed that the diameter of A-PTX-SF-NPs was higher than that of PTX-SF-NPs. The presence of the recombinant protein on the NP surface increased the particle size. Meanwhile, the surface charge of A-PTX-SF-NPs was more negative. The slight alteration may be due to the conjugation of the recombinant protein, which contained a large amount of aspartic acid and glutamic acid residues. ${ }^{19}$

In order to evaluate the cytotoxicity and targeting effect of A-PTX-SF-NPs, HeLa and MKN-45 cells were used as the cell models. We found that A-PTX-SF-NPs showed significantly increased cytotoxicity and targeting efficiency compared to PTX-SF-NPs in HeLa cells (Figures 3B and $4 \mathrm{~A}-\mathrm{C}$ ). In the MKN-45 cell line, the cell viability of the A-PTX-SF-NPs group was lower than that of PTX-SF-NPs group with no significant difference (Figure 3C). This slight difference may was attributed to the existence of iRGD on the surface of A-PTX-SF-NPs. It was also suggested that the biodistribution and therapeutic performance of NPs were closely related to their particle size. ${ }^{23}$ We speculated that the larger size of A-PTX-SF-NPs might be counteracting the function of iRGD partially.

We further examined the therapeutic efficiency of A-PTXSF-NPs in HeLa tumor-bearing nude mice. A-PTX-SF-NPs showed better antitumor effect than PTX-SF-NPs in vivo (Figure 5A), which was consistent with the in vitro cytotoxicity result. Having evaluated the in vivo antitumor efficiency of A-PTX-SF-NPs, in vivo tumor-homing ability was determined by NIR fluorescent imaging. A-PTX-SF-NPs and PTX-SF-NPs accumulated in the liver and intestine in the initial 1 hour post injection, indicating that some of the NPs were rapidly phagocytosed by the phagocytic cells and the reticuloendothelial system (RES). PTX-SF-NPs exhibited more tumor accumulation than A-PTX-SF-NPs at 4 hours post injection (Figure 6D). This phenomenon may be due to the smaller size of PTX-SF-NPs $(<150 \mathrm{~nm})$, leading to a better EPR effect. ${ }^{24}$ A-PTX-SF-NPs showed a higher level of tumor accumulation at the other detected time points, indicating that anti-EGFR-RGD might enhance the tumor-homing ability of NPs.

\section{Conclusion}

In this study, A-PTX-SF-NPs were prepared successfully by conjugation PTX-SF-NPs with anti-EGFR-iRGD. A-PTX-SF-NPs showed superior antitumor efficacy and better tumor targeting in vitro and in vivo compared to unconjugated NPs in EGFR overexpressing tumor cells. These results suggest that A-PTX-SF-NPs can be used as a potential carrier for targeted delivery in cancer therapy.

\section{Acknowledgments}

This work was supported by grants from the Natural Science Foundation of China (no 81272741, 81502037, and 81502029 ), the Natural Science Foundation of Jiangsu province (BK20150103), and the Medical Science and technology Foundation, Nanjing Department of Health (JQX 140001).

\section{Author contributions}

All authors contributed toward data analysis, drafting and critically revising the paper and agree to be accountable for all aspects of the work.

\section{Disclosure}

The authors declare no conflicts of interest in this work.

\section{References}

1. Sinha R, Kim GJ, Nie S, Shin DM. Nanotechnology in cancer therapeutics: bioconjugated nanoparticles for drug delivery. Mol Cancer Ther. 2006; 5(8):1909-1917.

2. Wang AZ, Langer R, Farokhzad OC. Nanoparticle delivery of cancer drugs. Anпu Rev Med. 2012;63:185-198.

3. Matsumura Y, Maeda H. A new concept for macromolecular therapeutics in cancer chemotherapy: mechanism of tumoritropic accumulation of proteins and the antitumor agent smancs. Cancer Res. 1986; 46(12 pt 1):6387-6392.

4. Nichols JW, Bae YH. EPR: evidence and fallacy. J Control Release. 2014;190:451-464.

5. Steichen SD, Caldorera-Moore M, Peppas NA. A review of current nanoparticle and targeting moieties for the delivery of cancer therapeutics. Eur J Pharm Sci. 2013;48(3):416-427.

6. Perez-Soler R. HER1/EGFR targeting: refining the strategy. Oncologist. 2004;9(1):58-67.

7. Creixell M, Bohórquez AC, Torres-Lugo M, Rinaldi C. EGFR-targeted magnetic nanoparticle heaters kill cancer cells without a perceptible temperature rise. ACS Nano. 2011;5(9):7124-7129.

8. Liu P, Li Z, Zhu M, et al. Preparation of EGFR monoclonal antibody conjugated nanoparticles and targeting to hepatocellular carcinoma. J Mater Sci Mater Med. 2010;21(2):551-556. 
9. Popovtzer R, Agrawal A, Kotov NA, et al. Targeted gold nanoparticles enable molecular CT imaging of cancer. Nano Lett. 2008;8(12): 4593-4596.

10. Sarkar S, Mazumdar A, Dash R, Sarkar D, Fisher PB, Mandal M. ZD6474 enhances paclitaxel antiproliferative and apoptotic effects in breast carcinoma cells. J Cell Physiol. 2011;226(2):375-384.

11. Koizumi F, Kanzawa F, Ueda Y, et al. Synergistic interaction between the EGFR tyrosine kinase inhibitor gefitinib ("Iressa") and the DNA topoisomerase I inhibitor CPT-11 (irinotecan) in human colorectal cancer cells. Int J Cancer. 2004;108(3):464-472.

12. Giovannetti E, Lemos C, Tekle C, et al. Molecular mechanisms underlying the synergistic interaction of erlotinib, an epidermal growth factor receptor tyrosine kinase inhibitor, with the multitargeted antifolate pemetrexed in non-small-cell lung cancer cells. Mol Pharmacol. 2008;73(4):1290-1300.

13. Sugahara KN, Teesalu T, Karmali PP, et al. Tissue-penetrating delivery of compounds and nanoparticles into tumors. Cancer Cell. 2009;16(6): $510-520$.

14. Sugahara KN, Teesalu T, Karmali PP, et al. Coadministration of a tumor-penetrating peptide enhances the efficacy of cancer drugs. Science. 2010;328(5981):1031-1035.

15. Ajani JA, Fairweather J, Dumas P, Patt YZ, Pazdur R, Mansfield PF. Phase II study of Taxol in patients with advanced gastric carcinoma. Cancer J Sci Am. 1997;4(4):269-274.
16. Park DC, Kim JH, Lew YO, Kim DH, Namkoong SE. Phase II trial of neoadjuvant paclitaxel and cisplatin in uterine cervical cancer. Gynecol Oncol. 2004;92(1):59-63.

17. Yao H-J, Ju R-J, Wang X-X, et al. The antitumor efficacy of functional paclitaxel nanomicelles in treating resistant breast cancers by oral delivery. Biomaterials. 2011;32(12):3285-3302.

18. Guo S, Huang L. Nanoparticles containing insoluble drug for cancer therapy. Biotechnol Adv. 2014;32(4):778-788.

19. Sha H, Zou Z, Xin K, et al. Tumor-penetrating peptide fused EGFR single-domain antibody enhances cancer drug penetration into $3 \mathrm{D}$ multicellular spheroids and facilitates effective gastric cancer therapy. J Control Release. 2015;200:188-200.

20. Wu P, Liu Q, Li R, et al. Facile preparation of paclitaxel loaded silk fibroin nanoparticles for enhanced antitumor efficacy by locoregional drug delivery. ACS Appl Mater Interfaces. 2013;5(23):12638-12645.

21. Chari RV. Targeted delivery of chemotherapeutics: tumor-activated prodrug therapy. Adv Drug Deliv Rev. 1998;31(1-2):89-104.

22. Wang X, Kaplan DL. Functionalization of silk fibroin with NeutrAvidin and biotin. Macromol Biosci. 2011;11(1):100-110.

23. Davis ME, Shin DM. Nanoparticle therapeutics: an emerging treatment modality for cancer. Nat Rev Drug Discov. 2008;7(9):771-782.

24. Liechty WB, Peppas NA. Expert opinion: responsive polymer nanoparticles in cancer therapy. Eur J Pharm Biopharm. 2012;80(2): 241-246.
OncoTargets and Therapy

\section{Publish your work in this journal}

OncoTargets and Therapy is an international, peer-reviewed, open access journal focusing on the pathological basis of all cancers, potential targets for therapy and treatment protocols employed to improve the management of cancer patients. The journal also focuses on the impact of management programs and new therapeutic agents and protocols on

\section{Dovepress}

patient perspectives such as quality of life, adherence and satisfaction The manuscript management system is completely online and includes a very quick and fair peer-review system, which is all easy to use. Visit http://www.dovepress.com/testimonials.php to read real quotes from published authors. 